\title{
Tumor Lysis Syndrome following a Single Dose of Nivolumab for Relapsed Small-Cell Lung Cancer
}

\author{
Sarah Maryon Hayes ${ }^{a} \quad$ Christine Wiese ${ }^{b} \quad$ Robert Schneidewend $^{b}$

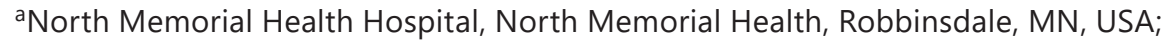 \\ ${ }^{b}$ North Memorial Health Cancer Center, North Memorial Health, Robbinsdale, MN, USA
}

\section{Keywords}

Immunotherapy · Small-cell lung cancer · Drug therapy

\begin{abstract}
Tumor lysis syndrome (TLS) is an oncologic emergency most frequently associated with initial treatment of hematologic malignancies and high-tumor burden solid tumors such as smallcell lung cancer ( $\mathrm{SCLC}$ ). Most often, TLS is associated with cytotoxic chemotherapy. In the treatment of SCLC, a paradigm shift has taken place in which immunotherapy is often added to chemotherapy for extensive-stage disease in the frontline setting or as monotherapy in the relapsed setting. Nivolumab is a programmed death 1 receptor blocking antibody previously FDA indicated for the treatment of metastatic SCLC with progression after platinum-based chemotherapy and at least one other line of therapy. Nivolumab, like all immune checkpoint inhibitor therapies, is associated with immune-mediated adverse reactions; however, there are few reported cases of nivolumab-induced TLS. We present a case of nivolumab-induced TLS following a single infusion. An 86-year-old female with a history of widely metastatic SCLC with metastasis to the liver, bone, and lymph nodes presented to the hospital following a fall due to weakness, dizziness, slurred speech, nausea, vomiting, and abdominal pain occurring 6 days after receiving her first nivolumab infusion. After extensive evaluation, the patient was diagnosed with TLS with hyperkalemia, acute renal failure, hyperphosphatemia, and hypocalcemia. She was treated aggressively with intravenous fluids, rasburicase, and sodium polystyrene sulfate (Kayexalate ${ }^{\circledR}$ ), which resulted in rapid improvement of her electrolytes and renal function. However, despite correction of electrolytes and overall symptomatic improvement, over the course of several days, the patient's condition rapidly deteriorated with increasing dyspnea, lethargy, confusion, and eventually death. TLS following nivolumab is exceedingly
\end{abstract}

\section{Karger}


rare. This report highlights the potential risk of development of TLS with checkpoint inhibitor therapy.

\section{Introduction}

Tumor lysis syndrome (TLS) is an oncologic emergency most frequently associated with the initial treatment of hematological malignancies, yet may occur in patients with solid tumors that are particularly sensitive to chemotherapy, such as small-cell lung cancer (SCLC). Rapid tumor cell death and lysis leads to spilling of intracellular contents into the bloodstream resulting in hyperuricemia, hyperphosphatemia, and hyperkalemia, which may be fatal due to the cardiac, nervous system, and renal toxicities that follow [1]. TLS in SCLC specifically is reported in the literature occurring spontaneously [2] prior to treatment or during initiation of treatment, especially in the setting of bulky disease and pretreatment hyperuricemia, elevated lactate dehydrogenase levels, or serum creatinine above the upper normal limit [3]. Twelve case reports of TLS in the setting of SCLC treatment were reported in a review, but these cases were all secondary to cytotoxic chemotherapy [4].

For decades, cytotoxic chemotherapy regimens comprising a platinum agent in combination with etoposide were the standard of care for extensive-stage SCLC, with a variety of cytotoxic therapies utilized in the relapsed setting. Nivolumab was previously listed in the SCLC National Comprehensive Cancer Network (NCCN) guidelines [5] as a category 2A treatment option for SCLC relapsed within 6 months of therapy in patients with an ECOG Performance Status of 0,1 , or 2 based on a phase 2 trial [6]. It was Food and Drug Administration (FDA) approved as monotherapy for extensive-stage SCLC treatment after progression on a platinum-containing regimen and a second regimen; however, the FDA-approval has since been withdrawn. Pembrolizumab monotherapy earned a category $2 \mathrm{~A}$ recommendation from NCCN in the same setting as nivolumab [7]. Checkpoint inhibitor-based chemoimmunotherapy regimens are now at the frontline of upfront SCLC treatment; atezolizumab or durvalumab in combination with cytotoxic chemotherapy are both category 1 (preferred) regimens for upfront treatment of extensive-stage SCLC [5]. As the role of immunotherapy continues to grow in the treatment of SCLC, it is vital to closely monitor postmarketing, realworld data to assess for risk of toxicities that may not fall in line with traditional immunemediated checkpoint inhibitor-related adverse events such as dermatitis, colitis, thyroiditis, and pneumonitis.

Case reports have previously described TLS with checkpoint blockade therapy in metastatic melanoma [8-10], metastatic renal cell carcinoma [11], widespread T-cell lymphoma [12], neuroendocrine cancer [13], metastatic urothelial carcinoma [14, 15], advanced hepatocellular carcinoma [16], and metastatic esophageal carcinoma [17] with mortality in most cases (shown in Table 1). One patient experienced grade 3 TLS in the phase 2 nivolumab (with or without ipilimumab) Checkmate-032 trial for SCLC [6]. TLS is an underreported complication of checkpoint blockade in the treatment of solid malignancies, as TLS may be misreported as acute kidney injury, renal failure, or electrolyte derangements [18]. Here, we describe a patient receiving nivolumab monotherapy for relapsed SCLC whose treatment was complicated by TLS and subsequent death after 1 immunotherapy infusion.

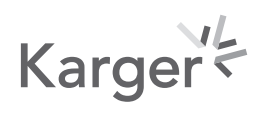


Table 1. Individual reports of tumor lysis syndrome following checkpoint inhibitor therapy

\begin{tabular}{lllll}
\hline Study & Cancer & Gender/age & Treatment & Doses, $n$ \\
\hline Brunnhoelzl and Wang [8] & Melanoma & $76 / \mathrm{M}$ & Nivolumab & 3 doses \\
Brunnhoelzl et al. [14] & Urothelial carcinoma & $77 / \mathrm{F}$ & Atezolizumab & 1 dose \\
Chan et al. [12] & NK/T-cell lymphoma & $80 / \mathrm{F}$ & Nivolumab & 2 doses \\
Chen [16] & Hepatocellular carcinoma & $65 / \mathrm{M}$ & Pembrolizumab & 1 dose \\
Fa'ak et al. [15] & Urothelial carcinoma & $67 / \mathrm{F}$ & Atezolizumab & 1 dose \\
Janjigian et al. [17] & Esophageal carcinoma & Unknown & Nivolumab plus & Unknown \\
& & & ipilimumab & \\
Masson Regnault et al. [9] & Melanoma & $73 / \mathrm{M}$ & Ipilimumab & 1 dose \\
Quadri [13] & Neuroendocrine tumor of & $52 / \mathrm{F}$ & Nivolumab plus & 1 dose \\
& the sigmoid colon & & ipilimumab & Nivolumab \\
Sater et al. [11] & Renal cell carcinoma & $74 / \mathrm{M}$ & dose \\
Sugimoto et al. [10] & Melanoma & $79 / \mathrm{M}$ & Nivolumab & 1 dose \\
\hline
\end{tabular}

\section{Case Report/Presentation}

An 86-year-old woman with a medical history including hypertension, hyperlipidemia, asthma, chronic obstructive pulmonary disease, idiopathic thrombocytic purpura, chronic kidney disease, lung adenocarcinoma of the right upper lobe, and small-cell carcinoma of the left upper lobe presented to the Emergency Department for evaluation of weakness, lower extremity edema, dizziness, and syncope. Her home medications included furosemide $40 \mathrm{mg}$ orally every morning, amlodipine $5 \mathrm{mg}$ orally every morning, gabapentin $100 \mathrm{mg}$ orally at bedtime, and as-needed senna, oxycodone, and ranitidine.

Her oncology history includes adenocarcinoma of the right upper lobe diagnosed in 2008 for which she underwent right upper lobectomy and mediastinal lymph node dissection with removal of mediastinal fat. She completed adjuvant chemotherapy treatment with 4 cycles of carboplatin and gemcitabine in June 2008 and declined radiation therapy. In October 2008, she developed a new spiculated left upper lobe mass and underwent stereotactic radiation. She continued surveillance without evidence of disease recurrence until September 2016, when she presented with a new hilar mass encasing the left upper lobe bronchus and a contralateral right upper lobe nodule. Following bronchoscopic evaluation, she was diagnosed with limited-stage small-cell carcinoma of the lung in September 2016 and underwent 4 cycles of palliative-intent (as the patient again declined radiation) carboplatin and etoposide therapy in June through December 2016; repeat scans showed stable-to-improved disease.

Four months later, she was found to have progressive disease in the left hilum, and she underwent palliative radiation therapy in May and June 2017. In late August 2017, she was found to have further progressive disease on imaging with metastasis to the liver, bones, and right upper lobe. Biopsy of the liver confirmed small-cell carcinoma. She subsequently underwent 4 cycles of carboplatin and etoposide starting in September 2017. Her treatment was complicated by severe fatigue and hospital admission for febrile neutropenia requiring significant dose reductions. Despite these complications, she had a very good response on imaging after the second cycle of therapy in October 2017. She completed treatment in December 2017 and was placed on observation to allow for recovery time following treatment.

Repeat scans in January 2018 showed osseous and hepatic progression of disease with lymphadenopathy and a new right upper lobe nodule. Comfort care approach versus immunotherapy was discussed with the patient, and a shared-decision was made to proceed with 
immunotherapy. Prior to initiating systemic therapy, she was hospitalized in January 2018 for left shoulder and upper back pain secondary to bony metastatic disease. She underwent radiation therapy to the cervical spine, but stopped in early February as she felt it was contributing to worsening heartburn.

She received the cycle 1, day 1 infusion of nivolumab 240 mg on February 21, 2018. On the day of treatment, she had significant bilateral lower extremity edema and a weeping left lower extremity wound. Her laboratory results showed leukocytosis (likely secondary to a week-long course of prednisone $20 \mathrm{mg}$ orally daily up until initiating nivolumab), worsening thrombocytopenia, hyponatremia, transaminitis, elevated BNP, and creatinine increased to $1.61 \mathrm{mg} / \mathrm{dL}$. Overall, the patient reported feeling well.

She presented to the Emergency Department 1 week following the nivolumab infusion complaining of weakness, bilateral lower extremity edema, nausea, slurred speech, and disorientation after collapsing while trying to get into her vehicle. Her electrocardiogram results showed tachycardia with T-wave abnormalities. She was afebrile with a blood pressure of 98/34 mm Hg and normal oxygen saturation on $2 \mathrm{~L}$ of oxygen via a nasal cannula. A chest $\mathrm{X}$-ray and head CT were obtained, and both were negative for acute abnormalities. Laboratory results indicated TLS with a serum uric acid level of $24.6 \mathrm{mg} / \mathrm{dL}$, potassium $6.8 \mathrm{mmol} / \mathrm{L}$, creatinine $3.61 \mathrm{mg} / \mathrm{dL}$, and phosphorus $7.4 \mathrm{mg} / \mathrm{dL}$ (shown in Fig. 1). The patient was given intravenous calcium gluconate, inhaled albuterol sulfate, intravenous regular insulin in combination with dextrose, sodium polystyrene sulfate $15 \mathrm{~g}$ orally, a fluid bolus of $500 \mathrm{~mL}$ $(9 \mathrm{~mL} / \mathrm{kg})$, and rasburicase $7.5 \mathrm{mg}$ intravenously. She was started on calcium acetate. Her labs improved the following day and as she was clinically improved, and she requested to return home. She was maintained as an inpatient to closely monitor her labs and to continue intravenous hydration.

On February 3, 2018, BMP showed normal sodium and potassium with improved uric acid, creatinine, calcium, $\mathrm{LDH}$, and phosphorus. WBC and hemoglobin remained stable, but platelets decreased further to $68 \mathrm{~K} / \mu \mathrm{L}$. Her peripheral blood smear showed reactive changes, no evidence of hemolysis, moderate normochromic normocytic anemia, mild leukocytosis with absolute neutrophilia, moderate thrombocytopenia, and leukoerythoblastic picture. She appeared weaker than the day before, with more labored breathing on oxygen via a nasal cannula, difficulty working with physical therapy due to profound fatigue, and worsening bilateral lower extremity edema due to fluids for management of TLS. At this time, she clinically appeared to be deteriorating rapidly, and thus hospice was recommended, for which the patient declined.

Overnight, she became more lethargic, confused, and dyspneic with hypoxia requiring BIPAP. Intravenous fluid resuscitation had to be countered with intravenous diuretics to attempt to improve her dyspnea and volume overload. Serum creatinine increased, and urine output declined. However, the patient was more alert the following morning and requested further discussion about treatment options. It was discussed that despite correction of the metabolic abnormalities from admission, physically she continued to decline and that a hospice approach would be reasonable. The family met with hospice care and later that afternoon officially transitioned to comfort cares. Through the day, her hypotension worsened, and she became more tachycardic and somnolent; unfortunately, the patient expired just hours later.

\section{Discussion}

To our knowledge, no postmarketing reports of TLS with checkpoint inhibitor treatment for SCLC have been published. This case adds to the growing number of case reports of TLS following checkpoint inhibitor therapy. Per Cairo-Bishop criteria for TLS [1], our patient met

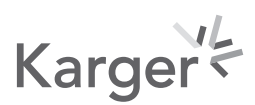




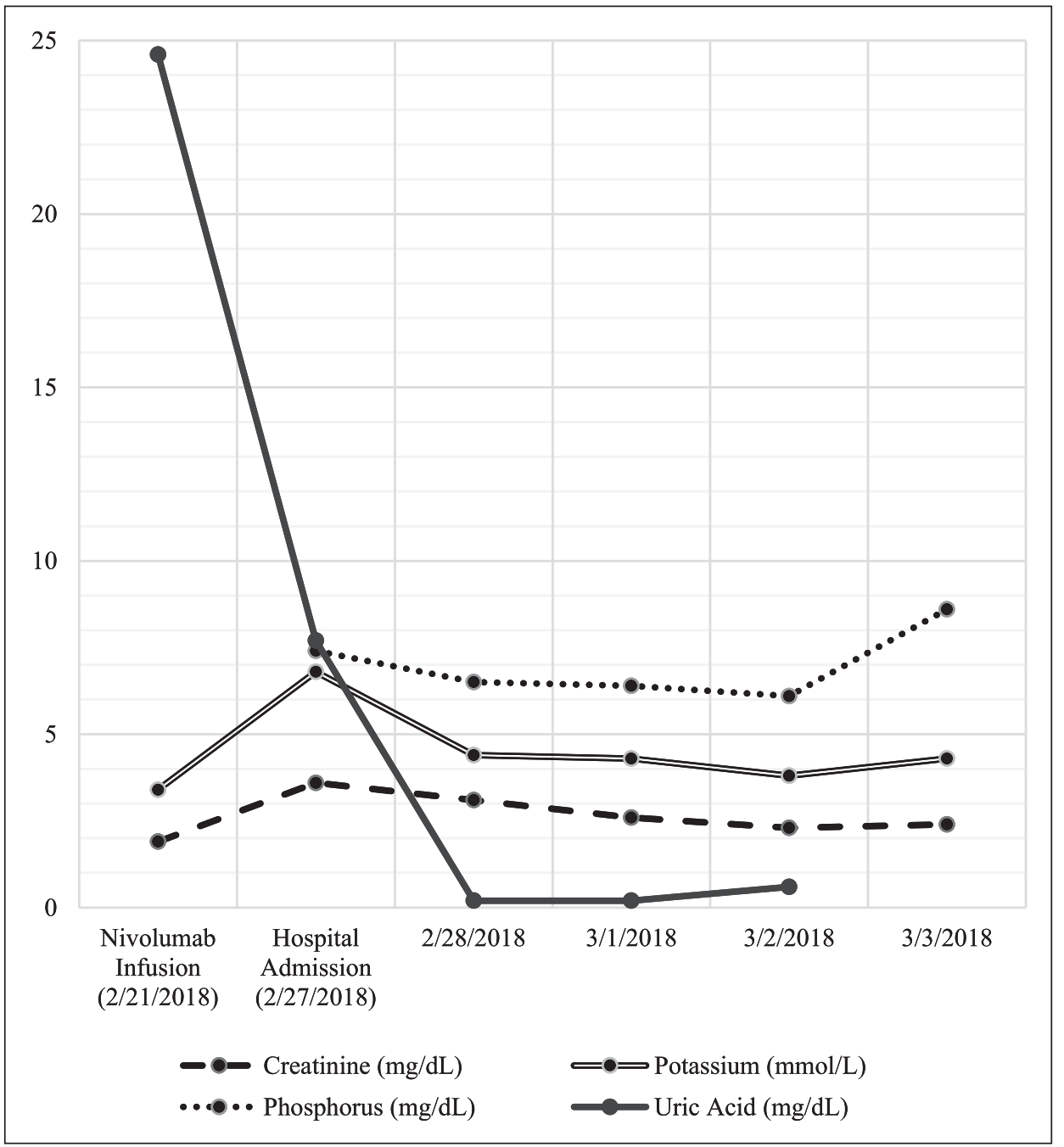

Fig. 1. Serum laboratory values relevant to tumor lysis syndrome at the time of infusion and during hospitalization.

3 of the 4 criteria for laboratory-defined TLS (uric acid $>8 \mathrm{mg} / \mathrm{dL}$, potassium $>6 \mathrm{mEq} / \mathrm{L}$, and phosphorus $>4.5 \mathrm{mg} / \mathrm{dL}$ ), occurring 6 days after the first nivolumab infusion.

Close vigilance and clinical monitoring for TLS in patients starting single-agent immunotherapy is not a routine clinical practice. Based on our patient's course and other reports published in the literature, we propose that clinicians consider empiric TLS monitoring in patients at high risk for TLS starting immunotherapy. Time-to-TLS is an important consideration for deciding when these patients need to be monitored. Our patient was in frank TLS just 6 days following the first infusion, but a wide range of timing has been described in case reports (2-33 days [18]). The timeframe in which TLS should be monitored for is made further unclear due to the delayed onset of response with immunotherapy, which may be months after treatment initiation.

Our patient had several risk factors for TLS, including bulky disease, high proliferation rate of tumor cells, and chronic kidney disease on outpatient diuretic therapy. Cordrey et al. [18] described characteristics of patients that experienced TLS associated with immunotherapy, including elderly age and liver metastases, both of which were common to our patient. Fewer than $10 \%$ of patients in CHECKMATE-032 with an SCLC diagnosis were older 
than 75 years; if elderly age is a risk factor for TLS development, it is possible that TLS may have been even rarer in this study population due to enrollment of few elderly patients. NCCN considers "active or previously documented autoimmune disease" as a contraindication to immunotherapy treatment. Our patient had a history of a single episode of idiopathic thrombocytic purpura in 2008 , treated with prednisone. Due to her autoimmune history, cytotoxic chemotherapy was considered instead of nivolumab, but ultimately nivolumab was chosen due to significant myelosuppression which warranted dose reductions with previous cytotoxic chemotherapy regimens. It is not currently known if patients with autoimmune conditions are more likely to develop immune-related adverse events with immunotherapy [19].

In addition to TLS, cytokine release syndrome (CRS) may have also been present in this case, especially due to her rapid neurological decline despite correction of electrolyte abnormalities. Laboratory data for D-dimer, fibrinogen, and bilirubin were not available to assist in establishing the CRS diagnosis. Checkpoint inhibitor-associated CRS appears at a median of 4 weeks after starting immunotherapy [20], making CRS less likely.

The majority of cancer diagnoses is in the elderly population and account for $80 \%$ of cancer deaths each year [21]. Age is one important patient factor in determining the appropriate cancer-directed therapy, including the use of immunotherapy. Elderly patients may experience unusual toxicities subsequent to checkpoint inhibitor administration with a possible increased risk [22]; in a subset analysis of several nivolumab trials, the rate of grade $3-5$ adverse effects was $71.7 \%$ in patients aged 70 years and older, compared with $58.4 \%$ in patients under 65 years old [23]. Frailty itself may serve as an indicator of the risk of adverse effects related to immunotherapy [21]. Elderly adults enrolled in clinical trials are often less frail and of better performance status than patients seen in clinical practice. As the cancer population continues to age, enrollment of elderly patients in clinical trials will help inform oncologists of the efficacy and safety of checkpoint inhibitor use in this population.

\section{Conclusion}

In summary, this case adds to the growing number of case reports of TLS as a complication of immunotherapy. Although TLS is not a classic or common sequela of checkpoint inhibitor therapy, this and other cases highlight the importance of monitoring for TLS following immunotherapy administration. TLS is rare, but possibly fatal; patient-specific features such as baseline bulky disease or renal insufficiency may help guide clinicians in knowing which patients to monitor with increased frequency.

\section{Acknowledgment}

There are no additional acknowledgments to be made for this manuscript.

\section{Statement of Ethics}

We ensure the accuracy, quality, and integrity of this case report. No identifying patient information was disclosed. Written consent for publication was obtained from the patient's next-of-kin on February 7, 2021. This manuscript was submitted to and reviewed by the North Memorial Health Institutional Review Board (exempt per Physician Research \& Education Coordinator).

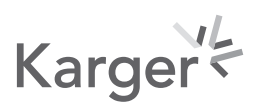




\section{Conflict of Interest Statement}

Dr. Sarah Maryon Hayes has no conflicts of interest to declare. Mrs. Christine Wiese has no conflicts of interest to declare. Dr. Robert Schneidewend has no conflicts of interest to declare.

\section{Funding Sources}

There were no funding sources pertaining to this publication, and no sponsorship was involved.

\section{Author Contributions}

Sarah Maryon Hayes contributed to literature review and manuscript writing. Christine Wiese contributed to manuscript writing, editing, and proofreading. Robert Schneidewend contributed to manuscript writing, editing, and proofreading.

\section{Data Availability Statement}

All data generated or analyzed during this study are included in this article. Further enquiries can be directed to the corresponding author.

\section{References}

1 Howard SC, Jones DP, Pui CH. The tumor lysis syndrome. N Engl J Med. 2011 May;364(19):1844-54.

2 Boonpheng B, Murtaza G, Ginn D. Spontaneous tumor lysis syndrome in a patient with metastatic small cell lung cancer: a case report. Case Rep Oncol. 2017 Apr;10(1):392-5.

3 Mott FE, Esana A, Chakmakjian C, Herrington JD. Tumor lysis syndrome in solid tumors. Support Cancer Ther. 2005 Apr;2(3):188-91.

4 Kanchustambham V, Saladi S, Patolia S, Stoeckel D. Spontaneous tumor lysis syndrome in small cell lung cancer. Cureus. 2017 Feb; 9(2):e1017.

5 National Comprehensive Cancer Network (NCCN). NCCN clinical practice guidelines in oncology. Small cell lung cancer. Version 3.2021. Fort Washington, PA: National Comprehensive Cancer Network; 2021.

6 Antonia SJ, Lopez-Martin JA, Bendell J, Ott PA, Taylor M, Eder JP, et al. Nivolumab alone and nivolumab plus ipilimumab in recurrent small-cell lung cancer (CheckMate 032): a multicenter, open-label, phase 1/2 trial. Lancet Oncol. 2016 Jul;17(7):883-95.

7 Chung HC, Lopez-Martin JA, Chuan-Hao Kao S, Miller WH, Ros W, Gao B, et al. Phase 2 study of pembrolizumab in advanced small-cell lung cancer (SCLC); KEYNOTE-158. J Clin Oncol. 2018 May;36(15):8506.

8 Brunnhoelzl D, Wang J. Acute tumor lysis after anti-pd-1 immunotherapy nivolumab for metastatic melanoma. J Mol Oncol Res. 2017;1(1):5-6.

9 Masson Regnault M, Ofaiche J, Boulinguez S, Tournier E, Rochaix P, Paul C, et al. Tumour lysis syndrome: an unexpected adverse event associated with ipilimumab. J Eur Acad Dermatol Venereol. 2017 Feb;31(2): e73-74.

10 Sugimoto S, Terashima T, Yamashita T, Iida N, Kitahara M, Hodo Y, et al. Tumor lysis syndrome in a patient with metastatic melanoma treated with nivolumab. Clin J Gastroenterol. 2020 Oct;13(5):935-9.

11 Sater HA, Patel RM, Sullivan BT, Parikh J. A case report of inflammatory syndrome presenting as tumor lysis syndrome after single dose of nivolumab. J Cancer Prev Curr Res. 2017 Jul;8(2):1-3.

12 Chan TSY, Li J, Loong F, Khong PL, Tse E, Kwong YL. PD1 blockade with low-dose nivolumab in NK/T cell lymphoma failing L-asparaginase: efficacy and safety. Ann Hematol. 2018 Jan;97(1):193-6.

13 Quadri S. Acute kidney injury from tumor lysis syndrome associated with ipilimumab/nivolumab therapy. Am J Kidney Dis. 2020 Apr;75(4):295.

14 Brunnhoelz D, Weed M, Trepet R, Wang J. Tumor lysis syndrome following a single atezolizumab infusion for metastatic urothelial carcinoma involving both upper and lower tract. Arch Can Res. 2017 Jan;5(1):1-5. 
15 Fa'ak F, Vanegas D, Osei KM. A case report of atezolizumab induced tumor lysis syndrome. Am J Case Rep. 2019 Jun;20:785-9.

16 Chen SC. Fever after anti-programmed cell death-1 treatment to predict the response in advanced hepatocellular carcinoma. J Clin Oncol. 2018 Feb;36(Suppl 5):90.

17 Janjigian YY, Bendell J, Calvo E, Kim JW, Ascierto PA, Sharma P, et al. CheckMate-032 study: efficacy and safety of nivolumab and nivolumab plus ipilimumab in patients with metastatic esophagogastric cancer. J Clin Oncol. 2018 Oct;36(28):2836-44.

18 Cordrey EO, Wang J. Tumor lysis syndrome associated with immune checkpoint blockade in solid tumors. Jpn J Cancer Oncol Res. 2018 Jun;1(1):1005.

19 Coureau M, Meert AP, Berghmans T, Grigoriu B. Efficacy and toxicity of immune -checkpoint inhibitors in patients with preexisting autoimmune disorders. Front Med. 2020 May; 7:137.

20 Ceschi A, Noseda R, Palin K, Verhamme K. Immune checkpoint inhibitor-related cytokine release syndrome: analysis of WHO global pharmacovigilance database. Front Pharmacol. 2020 May;11:557.

21 Ethun CG, Bilen MA, Jani AB, Maithel SK, Ogan K, Master VA. Frailty and cancer: implications for oncology surgery, medical oncology, and radiation oncology. CA Cancer J Clin. 2017 Sept;67(5):362-77.

22 Elias R, Odejide O. Immunotherapy in older adults: a checkpoint to palliation? Am Soc Clin Oncol Educ Book. 2019 Jan;39:e110-20.

23 Singh H, Kim G, Maher VE, Beaver JA, Pai-Scherf LH, Balasubramaniam S, et al. FDA subset analysis of the safety of nivolumab in elderly patients with advanced cancers. J Clin Oncol. 2016;34(Suppl 15):10010. 